\title{
On the growth of perturbations in interacting dark energy and dark matter fluids.
}

\author{
N.A. Koshelev \\ Received: date / Accepted: date

\begin{abstract}
The covariant generalizations of the background dark sector coupling suggested in G. Mangano, G. Miele and V. Pettorino, Mod. Phys. Lett. A 18, 831 (2003) are considered. The evolution of perturbations is studied with detailed attention to interaction rate that is proportional to the product of dark matter and dark energy densities. It is shown that some classes of models with coupling of this type do not suffer from early time instabilities in strong coupling regime.
\end{abstract}

Keywords Cosmology

PACS $98.80 . \mathrm{Cq} \cdot 95.35 .+\mathrm{d} \cdot 95.36 .+\mathrm{x}$

\section{Introduction}

Recent observations have shown that the Universe is spatially flat and presently accelerating. Most attempts to explain this acceleration involve the introduction of dark energy, as a source in the Einstein field equations. In addition, the important contribution to the total density is the dark matter, whose existence is inferred indirectly by observing its gravitational influence on normal matter, such as stars, gas and dust. Standard $\Lambda C D M$ model, in which dark energy is considered as a small positive cosmological constant and dark matter treated as gas of cold non-baryonic particles, provides a very good fit to the supernovae data [1] as well as CMB measurements [2] and observations of large scale structure [3], but the small and fine-tuned value of the cosmological constant cannot be explained within current particle physics [4]. As a result a lot of other more expected from theoretical point of view cosmological models have been proposed to giving a dynamical origin to dark energy.

Models with non-minimal coupling of dark matter and dark energy have called attention in the last decade. The nature of both dark energy and dark matter are still unknown, and in principle, the additional interaction between them is possible. Moreover, the coupling between matter and quintessence is motivated by high energy particle physics considerations (see [5] for a set of references). There are a large number of works in where the interaction is seen in models with quintessence [5,6,7, 8, 9, 10, 11, 12] field. Also available the

Ulyanovsk State University, Leo Tolstoy str 42, 432970, Russia

E-mail: koshna71@inbox.ru 
phenomenological approach, in which the interaction is introduced into the conservation equations for dark matter $(c)$ and dark energy $(x)$, considered as perfect fluids. The background energy exchange in the dark sector can be represented by

$$
\begin{gathered}
\bar{\rho}_{c}^{\prime}=-3 \mathscr{H} \bar{\rho}_{c}+a \bar{Q}, \\
\bar{\rho}_{x}^{\prime}=-3 \mathscr{H}\left(1+w_{x}\right) \bar{\rho}_{x}-a \bar{Q},
\end{gathered}
$$

where the prime denotes derivative with respect to conformal time $\tau$, the bars mark the background quantities, $a$ is the scale factor, $\mathscr{H}=a^{\prime} / a, w_{x}=\bar{P}_{x} / \bar{\rho}_{x}$ and the coupling $\bar{Q}$ is some function of background variables.

In the simplest models the quantity $\bar{Q}$ is a linear combination of the dark sector densities

$$
\bar{Q}=A_{c} \bar{\rho}_{c}+A_{x} \bar{\rho}_{x},
$$

where $A_{I}=3 \alpha_{I} H$ or $A_{I}=\Gamma_{I}$. Here $\alpha_{I}$ are the dimensionless constants and $\Gamma_{I}$ are constant interaction rates [13]. There are a large number of works in which for coupling (3) was considered the background [13, 14, 15] and the perturbations evolution [16, 17, 18, 19, 20, 21].

In the paper [22] in the context of the stimulated decay of dark energy into dark matter had been proposed the interaction of the form

$$
\bar{Q}=\gamma \bar{\rho}_{c}^{\alpha} \bar{\rho}_{x}^{\beta},
$$

where $\alpha, \beta$ are the dimensionless constants, and $\gamma$ is a constant parameter with dimension $\left[\right.$ density $^{1-\alpha-\beta} \times$ time $\left.^{-1}\right]$. This coupling provide a mechanism to alleviate the cosmic coincidence problem [23], [24], namely why the dark energy density nearly coincides with the dark matter density presently. The models with interaction (4) also have been studied in the framework of holographic dark energy [25]. By assuming that $\alpha=1, \beta=0$ or $\alpha=0, \beta=1$ one obtains the particular cases of coupling (3).

Although the basic properties of background solutions for the interaction (4) are previously considered, the perturbation evolution in such scenarios is not examined except the mentioned above special cases. In this paper we shall cover the perturbation equations and investigate the solutions of them with detailed attention to the most physically reasonable choice of parameters $\alpha=\beta=1$.

\section{Background.}

We consider the spatially flat Universe filled with radiation $(r)$, massless neutrino $(v)$, baryonic matter $(b)$, cold dark matter $(c)$ and dark energy $(x)$. We choose the linear parametrization of the dark energy equation of state [26]

$$
w_{x}(a)=w_{0}+w_{1}(1-a)
$$

with constants $w_{0}>-1$ and $0<w_{1}<-w_{0}$, so the dark energy is subdominant with respect to the radiation at early times and the phantom divide line $w_{x}=-1$ crossing does not occur. Since $w_{x}^{\prime} / \mathscr{H}=-a w_{1}$, in the radiation dominated era the dark energy equation of state parameter $w_{x}$ is well approximated by a constant $w_{0}+w_{1}$.

Background dynamics in the presence of coupling (4) is completely described by Friedmann's equation

$$
\mathscr{H}^{2}=\frac{8 \pi G}{3} a^{2} \bar{\rho}
$$



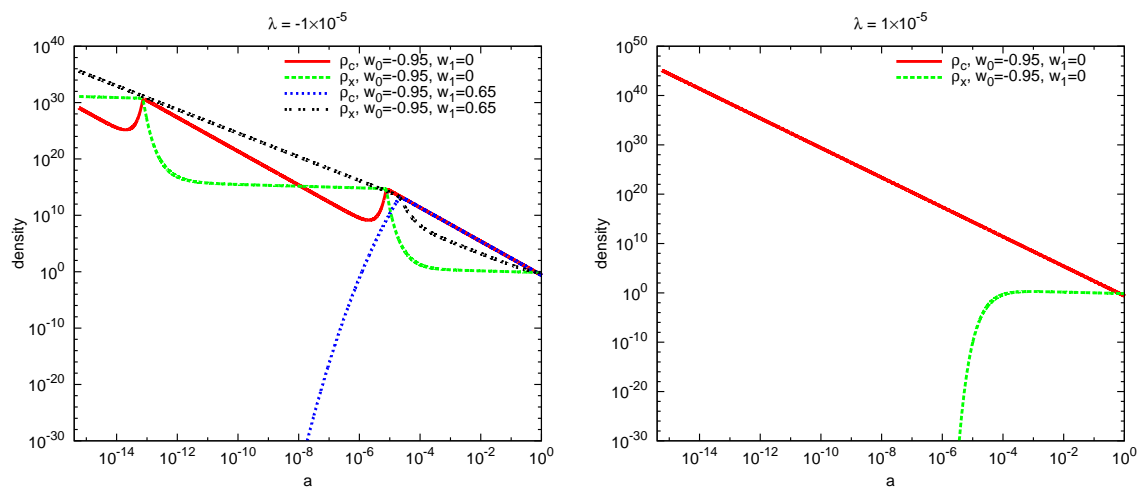

Fig. 1 Background energy densities in units of $\rho_{0 c r}$ for $\alpha=\beta=1, H_{0}=70 \mathrm{~km} \mathrm{~s}^{-1} \mathrm{Mpc}^{-1}, \Omega_{x}=0.70$, $\Omega_{c}=0.2538, \Omega_{b}=0.0462$. Examples of dark sector densities evolution at negative and positive coupling are shown on left and right panels, respectively.

and continuity equations

$$
\begin{gathered}
\bar{\rho}_{r}^{\prime}=-4 \mathscr{H} \bar{\rho}_{r}, \quad \bar{\rho}_{v}^{\prime}=-4 \mathscr{H} \bar{\rho}_{v}, \\
\bar{\rho}_{b}^{\prime}=-3 \mathscr{H} \bar{\rho}_{b}, \\
\bar{\rho}_{c}^{\prime}=-3 \mathscr{H} \bar{\rho}_{c}-a \gamma \bar{\rho}_{c}^{\alpha} \bar{\rho}_{x}^{\beta}, \\
\bar{\rho}_{x}^{\prime}=-3 \mathscr{H}\left(1+w_{x}\right) \bar{\rho}_{x}+a \gamma \bar{\rho}_{c}^{\alpha} \bar{\rho}_{x}^{\beta} .
\end{gathered}
$$

To determines the characteristic strength of the dark sector coupling it is convenient to introduce the dimensionless parameter $\lambda$ defined by [25]

$$
\lambda=\gamma \rho_{0 c r}^{\alpha+\beta-1} H_{0}^{-1}
$$

where $\rho_{0 c r}=3 H_{0}^{2} /(8 \pi G)$ is the current critical density.

System of equations (6)-(10) can be numerically solved, as was done in [22] for $\alpha=1$ and the dark energy equation of state $w_{x}=-1$. However, the equations (9) and (10) immediately suggest several analytical conclusions.

When $\gamma<0$, the dark energy density drops with the scale factor $a(\tau)$ not slower than $a^{-3\left(1+w_{0}\right)}$ and the dark matter density can increase under $\left|a \gamma \bar{\rho}_{c}^{\alpha-1} \bar{\rho}_{x}^{\beta} / \mathscr{H}\right|>3$ condition. These features offer a simple way out to the cosmic coincidence problem, since in this models the present situation of $\bar{\rho}_{x} \sim \bar{\rho}_{c}$ have occurred many times in the past [22].

The class of models with positive $\gamma$ also describe a interesting scenarios, as it allow to consider the dark energy grew out due the dark matter decay. However, the interaction of this type potentially leads to unphysical results, because at early times the dark energy density could be driven to negative value in some models with decaying dark matter [16].

Figure 1 shows the time evolution of the dark matter and dark energy densities at values parameters $\alpha=\beta=1$. The pattern of the solutions varies depending on the model parameters. When $w_{x}<-1 / 3$ at high redshifts and $\gamma<0$, one can obtain a sequence of regimes of the weak

$\max \left(\left|a \gamma \bar{\rho}_{c} / \mathscr{H}\right|,\left|a \gamma \bar{\rho}_{x} / \mathscr{H}\right|\right) \ll 1$ and strong $\max \left(\left|a \gamma \bar{\rho}_{c} / \mathscr{H}\right|,\left|a \gamma \bar{\rho}_{x} / \mathscr{H}\right|\right) \geq 1$ dark sector coupling. At the stage of the weak interaction under the condition $w_{x}<-1 / 3$ the dark energy density decays more slowly than $a^{-2}(\tau)$, and hence in the radiation dominated era the 
inequality $\left|a \gamma \bar{\rho}_{x} / \mathscr{H}\right|>3$ will be reached at some time, from which the density of dark matter increases. The increased dark matter density increases the dark energy decay rate, the condition $\left|a \gamma \bar{\rho}_{x} / \mathscr{H}\right| \ll 1$ recovers and dark energy has no longer significant impact on the evolution of dark matter. Moreover, since the dark matter density is now reduced proportional to the inverse third degree of the scale factor, there comes a point when the influence of dark matter to dark energy also becomes negligible, i.e. a next stage of a weak interaction begins. For $-1 / 3<w_{x}$ however, if the interaction is weak initially, it will remain so through the subsequent evolution. This means that the stage of strong interaction in such models is only one. Accordingly, in such models the density of dark matter in the radiation dominated era is extremely small.

The right panel of Figure 1 depicts the time evolution of the density of dark matter and dark energy at values parameters $\alpha=\beta=1$ and positive $\gamma$. In this case, the density of dark energy is always positive.

\section{Model and perturbed equations.}

Let us consider a spatially flat FLRW Universe with small scalar type perturbations. The line element may be written as

$$
d s^{2}=a^{2}(\tau)\left\{-(1+2 \phi) d \tau^{2}+2 B_{, i} d \tau d x^{i}+\left[(1-2 \psi) \delta_{i j}+2 E_{, i j}\right] d x^{i} d x^{j}\right\} .
$$

The energy-momentum tensor of a perfect $A$-fluid is given by

$$
T_{A v}^{\mu}=\left(\rho_{A}+P_{A}\right) u_{A}^{\mu} u_{A v}+P_{A} \delta_{v}^{\mu},
$$

where $\rho_{A}=\bar{\rho}_{A}\left(1+\delta_{A}\right)$ is density, $P_{A}=\bar{P}_{A}+\delta P_{A}$ is pressure, and the fluid four-velocity $u_{A}^{\mu}=d x_{A}^{\mu} / d s$ at linear order of the perturbations is

$$
u_{A}^{\mu}=\frac{1}{a}\left[(1-\phi), v_{A}^{, i}\right], \quad u_{v}=a\left[-(1+\phi), v_{A, i}+B_{, i}\right] .
$$

We assume that the anisotropic stress of dark energy and dark matter vanishes, so the dark species can be treated as perfect fluids. In the general case of coupling fluids divergence of the energy-momentum tensor of each fluid yields

$$
T_{A ; v}^{\mu v}=Q_{A}^{\mu},
$$

where 4-vectors $Q_{A}^{\mu}$ are restricted by the constraint [27]

$$
\sum_{A} Q_{A}^{\mu}=0
$$

which follow from the conservation law of the total energy-momentum tensor. For convenience one can decompose these 4 -vectors into two parts

$$
Q_{A}^{\mu}=Q_{A} u^{\mu}+F_{A}^{\mu}, \quad Q_{A}=\bar{Q}_{A}+\delta Q_{A}, \quad u_{\mu} F_{A}^{\mu}=0 .
$$

Here $u^{\mu}$ is the total four-velocity, $Q_{A}$ is the energy density transfer rate and $F_{A}^{\mu}$ is the momentum density transfer rate of $A$-fluid in the total matter gauge. By definition, $F_{A}^{\mu}=a^{-1}\left(0, f_{A}{ }^{, i}\right)$, where $f_{A}$ is a momentum transfer potential [16]. 
The energy exchange in the background does not determine the covariant form of energy exchange. Instead, an energy exchange four-vectors $Q_{A}^{\mu}$ must be specified. The simplest scalars, which can be used to construct quantities $Q_{c}^{\mu}, Q_{x}^{\mu}$ are

$$
T_{c \sigma}^{\sigma}=\rho_{c}, \quad T_{x \sigma}^{\sigma}=\rho_{x}-3 p_{x}, \quad T_{c v}^{\sigma} T_{x \sigma}^{v}=\rho_{c} \rho_{x}
$$

where the last relation holds at linear order. Accordingly, the direct generalization (4) with

$$
Q_{c}=-Q_{x}=-\gamma \rho_{c}^{\alpha} \rho_{x}^{\beta},
$$

is covariant at the first order.

We are interested first of all in models with decaying dark matter or with decaying dark energy. The most natural way is to choose a vector $F_{A}^{\mu}$ in the form

$$
F_{c}^{\mu}=-F_{x}^{\mu}=\frac{1}{a}\left(0,\left(\gamma \rho_{c}^{\alpha} \rho_{x}^{\beta}\left(v-v_{c}\right)\right)^{, i}\right)
$$

or

$$
F_{c}^{\mu}=-F_{x}^{\mu}=\frac{1}{a}\left(0,\left(\gamma \rho_{c}^{\alpha} \rho_{x}^{\beta}\left(v-v_{x}\right)\right)^{, i}\right) .
$$

The momentum transfer vanishes in the dark matter rest frame or in the dark energy rest frame respectively. A few more general form of coupling can be written as

$$
Q_{x}^{\mu}=-Q_{c}^{\mu}=\rho_{c}^{\alpha} \rho_{x}^{\beta}\left(\gamma_{c} u_{c}^{\mu}+\gamma_{x} u_{x}^{\mu}\right),
$$

where $\gamma_{c}$ and $\gamma_{x}$ are arbitrary constants.

The continuity equations for coupled perfect fluids can be obtained by linearization of equations (15). As a result, one can write [16]

$$
\begin{gathered}
\delta \rho_{A}^{\prime}+3 \mathscr{H}\left(\delta \rho_{A}+\delta P_{A}\right)-3\left(\bar{\rho}_{A}+\bar{P}_{A}\right) \psi^{\prime}-k^{2}\left(\bar{\rho}_{A}+\bar{P}_{A}\right)\left(v_{A}+E^{\prime}\right)=a \bar{Q}_{A} \phi+a \delta Q_{A}, \\
{\left[\left(\bar{\rho}_{A}+\bar{P}_{A}\right)\left(v_{A}+B\right)\right]^{\prime}+4 \mathscr{H}\left(\bar{\rho}_{A}+\bar{P}_{A}\right)\left(v_{A}+B\right)+\left(\bar{\rho}_{A}+\bar{P}_{A}\right) \phi+\delta P_{A}=a \bar{Q}_{A}(v+B)+a f_{A},}
\end{gathered}
$$

where for coupling (22)

$$
\begin{gathered}
\delta Q_{c}=-\delta Q_{x}=-\left(\gamma_{c}+\gamma_{x}\right) \bar{\rho}_{c}^{\alpha} \bar{\rho}_{x}^{\beta}\left(\alpha \delta_{c}+\beta \delta_{x}\right), \\
f_{c}=-f_{x}=\bar{\rho}_{c}^{\alpha} \bar{\rho}_{x}^{\beta}\left(\gamma_{c}\left(v-v_{c}\right)+\gamma_{x}\left(v-v_{x}\right)\right) .
\end{gathered}
$$

Pressure perturbations in the general case can be expressed in terms of the density perturbations and the velocity potentials

$$
\delta P_{A}=c_{s A}^{2} \delta \rho_{A}+\left(c_{s A}^{2}-c_{s A(a d)}^{2}\right)\left(3 \mathscr{H}\left(1+w_{A}\right) \rho_{A}-a \bar{Q}_{A}\right) \frac{\theta_{A}}{k^{2}} .
$$

where $\theta_{A}=-k^{2}\left(B+v_{A}\right), c_{A s(a d)}^{2}=P_{A}^{\prime} / \rho_{A}^{\prime}$ is the adiabatic sound speed and the rest frame sound speed $s_{S A}$ defined by

$$
c_{s A}^{2}=\left.\frac{\delta P_{A}}{\delta \rho_{A}}\right|_{A-\text { fluid rest frame }} .
$$

We will work in synchronous orthogonal gauge $\phi=0, B=0$ in the Fourier space, using the notation of [28]:

$$
\psi_{\mathbf{k}}=\eta, \quad k^{2} E_{\mathbf{k}}=-h / 2-3 \eta .
$$


Now the conservation equations (15) for the dark energy and dark matter in the synchronous gauge takes the form

$$
\begin{gathered}
\delta_{x}^{\prime}+3 \mathscr{H}\left(c_{s x}^{2}-w_{x}\right) \delta_{x}+\left(1+w_{x}\right) \theta_{x}+3 \mathscr{H}\left[3 \mathscr{H}\left(1+w_{x}\right)\left(c_{s x}^{2}-w_{x}\right)+w_{x}^{\prime}\right] \frac{\theta_{x}}{k^{2}} \\
+\frac{1+w_{x}}{2} h^{\prime}=\left(\gamma_{c}+\gamma_{x}\right) a \bar{\rho}_{c}^{\alpha} \bar{\rho}_{x}^{\beta-1}\left[\alpha \delta_{c}+(\beta-1) \delta_{x}+3 \mathscr{H}\left(c_{s x}^{2}-w_{x}\right) \frac{\theta_{x}}{k^{2}}\right] \\
\delta_{c}^{\prime}+\frac{1}{2} h^{\prime}+\theta_{c}=\left(\gamma_{c}+\gamma_{x}\right) a \bar{\rho}_{c}^{\alpha-1} \bar{\rho}_{x}^{\beta}\left[(1-\alpha) \delta_{c}-\beta \delta_{x}\right] \\
\theta_{x}^{\prime}+\mathscr{H}\left(1-3 c_{s x}^{2}\right) \theta_{x}-\frac{c_{s x}^{2} k^{2}}{1+w_{x}} \delta_{x}=\frac{a \bar{\rho}_{c}^{\alpha} \bar{\rho}_{x}^{\beta-1}}{1+w_{x}}\left(\gamma_{c}\left(\theta_{c}-\theta_{x}\right)-\left(\gamma_{c}+\gamma_{x}\right) c_{s x}^{2} \theta_{x}\right), \\
\theta_{c}^{\prime}+\mathscr{H} \theta_{c}=\gamma_{x} a \bar{\rho}_{c}^{\alpha-1} \bar{\rho}_{x}^{\beta}\left[\theta_{c}-\theta_{x}\right] .
\end{gathered}
$$

Note that in this phenomenological approach the sound speed $c_{s x}$ is needed to be fixed by hand [29]. In the case of quintessence dark energy one have to set $c_{s x}=1$, and we adopt this value in the following.

The perturbed Einstein equations are well known, and can be found in [28]. We reproduce here only one of them

$$
h^{\prime \prime}+\mathscr{H} h^{\prime}=-8 \pi G^{2} a^{2}(\delta \rho+3 \delta P) .
$$

Equations (30) - 33) provide a set of coupled equations covering the dark sector density evolution.

\section{Large scale perturbations.}

The coupling terms appearing in the dark energy pressure perturbations may lead to the early time instabilities, as was first pointed out in [16]. The similar phenomenon is well known in inflationary multi-fields models, where on large scales entropy perturbations can source adiabatic ones [30]. To study this phenomenon here we write the second order differential equations for the dark energy density perturbations. This approach [18] allows to identify areas of possible instabilities before solving the perturbation equations.

The perturbed fluid equations (30) - 32) and equation (34) can be combined to

$$
\delta_{x}^{\prime \prime}+A_{x} \mathscr{H} \delta_{x}^{\prime}+B_{x} \mathscr{H}^{2} \delta_{x}=C_{x} \mathscr{H}^{2},
$$

where on large scales $(k \ll \mathscr{H})$

$$
\begin{array}{r}
A_{x}=1-3 w_{x}-\frac{2 \mathscr{H}^{\prime}}{\mathscr{H}^{2}}+\left(\frac{\gamma_{c}+\gamma_{+}}{1+w_{x}}-(\beta-1) \gamma_{+}\right) \frac{a \bar{\rho}_{c}^{\alpha} \bar{\rho}_{x}^{\beta-1}}{\mathscr{H}}-f \\
B_{x}=3\left(1-w_{x}\right)\left(1-\frac{\mathscr{H}^{\prime}}{\mathscr{H}^{2}}+\frac{\gamma_{c} a \bar{\rho}_{c}^{\alpha} \bar{\rho}_{x}^{\beta-1}}{\left(1+w_{x}\right) \mathscr{H}}-f\right)-\frac{3 w_{x} w_{x}^{\prime}}{\left(1+w_{x}\right) \mathscr{H}} \\
+(\beta-1) \gamma_{+} \frac{a \bar{\rho}_{c}^{\alpha} \bar{\rho}_{x}^{\beta-1}}{\mathscr{H}}\left(2+\frac{\mathscr{H}^{\prime}}{\mathscr{H}^{2}}-\frac{\gamma_{c}+\gamma_{+}}{1+w_{x}} \frac{a \bar{\rho}_{c}^{\alpha} \bar{\rho}_{x}^{\beta-1}}{\mathscr{H}}+f\right) \\
\quad-(\beta-1) \gamma_{+} \frac{1}{\mathscr{H}}\left(\frac{a \bar{\rho}_{c}^{\alpha} \bar{\rho}_{x}^{\beta-1}}{\mathscr{H}}\right)^{\prime}+\gamma_{+}^{2} \alpha \beta \frac{a^{2} \bar{\rho}_{c}^{2 \alpha-1} \bar{\rho}_{x}^{2 \beta-1}}{\mathscr{H}^{2}}
\end{array}
$$




$$
\begin{array}{r}
C_{x}=\frac{1}{2}\left(1+w_{x}\right)\left(3+\frac{2 \mathscr{H}^{\prime}}{\mathscr{H}^{2}}-\frac{\gamma_{c}+\gamma_{+}(\alpha+1)}{1+w_{x}} \frac{a \bar{\rho}_{c}^{\alpha} \bar{\rho}_{x}^{\beta-1}}{\mathscr{H}}+f\right) \frac{h^{\prime}}{\mathscr{H}}-\frac{w_{x}^{\prime} h^{\prime}}{2 \mathscr{H}^{2}} \\
-3 \mathscr{H}\left(3-3 w_{x}+\frac{w_{x}^{\prime}}{\left(1+w_{x}\right) \mathscr{H}}-\frac{1-w_{x}}{1+w_{x}} \frac{\gamma_{+} a \bar{\rho}_{c}^{\alpha} \bar{\rho}_{x}^{\beta-1}}{\mathscr{H}}\right) \frac{\gamma_{c} a \bar{\rho}_{c}^{\alpha} \bar{\rho}_{x}^{\beta-1}}{\mathscr{H}} \frac{\theta_{c}}{k^{2}} \\
+\frac{\gamma_{+}}{\mathscr{H}}\left(\frac{a \bar{\rho}_{c}^{\alpha} \bar{\rho}_{x}^{\beta-1}}{\mathscr{H}}\right)^{\prime} \alpha \delta_{c}-\left(2+\frac{\mathscr{H}^{\prime}}{\mathscr{H}^{2}}-\frac{\gamma_{c}+\gamma_{+}}{1+w_{x}} \frac{a \bar{\rho}_{c}^{\alpha} \bar{\rho}_{x}^{\beta-1}}{\mathscr{H}}+f\right) \frac{\gamma_{+} a \bar{\rho}_{c}^{\alpha} \bar{\rho}_{x}^{\beta-1}}{\mathscr{H}} \alpha \delta_{c} \\
+(1-\alpha) \frac{\gamma_{+}^{2} a^{2} \bar{\rho}_{c}^{2 \alpha-1} \bar{\rho}_{x}^{2 \beta-1}}{\mathscr{H}^{2}} \alpha \delta_{c}+\frac{3}{2}\left(1+w_{x}\right)\left(\delta+3 \frac{\delta P}{\rho}\right),
\end{array}
$$

and

$$
f=\frac{1}{\mathscr{H}}\left[\ln \left|\left(1-w_{x}\right)\left(3+3 w_{x}-\frac{\gamma_{+} a \bar{\rho}_{c}^{\alpha} \bar{\rho}_{x}^{\beta-1}}{\mathscr{H}}\right)+\frac{w_{x}^{\prime}}{\mathscr{H}}\right|\right]^{\prime}, \gamma_{+}=\gamma_{c}+\gamma_{x}
$$

In case of minimal coupling and a constant dark energy equation of state the quantity $f$ is zero at all times. When $\gamma_{c}=0$ or $\gamma_{x}=0$, terms with $\theta_{c}$ can be ignored, since in the latter case one can work in particular synchronous orthogonal gauge in which the dark matter fluid has a vanishing velocity.

The equation (35) is written in slightly different form then was discussed in the Ref. [18]. In particular, it involves $h^{\prime}$ instead of the time derivative of dark matter density perturbations. This difference can be valuable, since both quantities $h^{\prime}, \delta_{c}^{\prime}$ are related by equation (31) and, in the general case, may implicitly depend on the dark energy perturbations. Used here notation is convenient to study of generations of non-adiabatic perturbations in the radiation dominated era.

At first approximation, the source terms can be calculated using usual adiabatic mode solutions. Since the dark species are subdominant in early Universe, the corresponding contributions to $h^{\prime}$ and total $\delta, \delta P$ are negligible. Actually, this is the assumption about the initial conditions, but it is certainly satisfied if the initial adiabatic conditions are imposed at the stage of weak coupling. This assumption breaks down if the dark energy perturbations have increased dramatically. It means that the right hand side of the equation (35) can be treated at early times and on initial stages of dark energy inhomogeneities growth as an external force that is independent of the dark energy perturbations. In this approach, the negative sign of $A_{x}, B_{x}$ or both of them indicate on the existence of large scale instabilities due a anti-damping force or exponential growth of intrinsic dark energy perturbations. The nature of instability can be revealed by considering the time evolution of the gauge-invariant curvature perturbation on uniform density hypersurfaces

$$
\zeta \equiv-\psi-\mathscr{H} \frac{\delta \rho}{\bar{\rho}}
$$

that is conserved on large scales for adiabatic perturbations.

We carry out the detailed analysis of perturbations for two cases: $\gamma_{c}=\gamma, \gamma_{x}=0$ and $\gamma_{c}=0, \gamma_{x}=\gamma$ with $\alpha=\beta=1$, i.e. for

$$
Q_{x}^{\mu}=-Q_{c}^{\mu}=\gamma \rho_{c} \rho_{x}\left(b u_{c}^{\mu}+(1-b) u_{x}^{\mu}\right)
$$

where $b=1$ for the first case and $b=0$ for the second. 
For coupling above

$$
\begin{gathered}
A_{x}=1-3 w_{x}-\frac{2 \mathscr{H}^{\prime}}{\mathscr{H}^{2}}+\frac{b+1}{1+w_{x}} \frac{\gamma a \bar{\rho}_{c}}{\mathscr{H}}-f, \\
B_{x}=3\left(1-w_{x}\right)\left(1-\frac{\mathscr{H}^{\prime}}{\mathscr{H}^{2}}+b \frac{\gamma a \bar{\rho}_{c}}{\left(1+w_{x}\right) \mathscr{H}}-f\right)-\frac{3 w_{x} w_{x}^{\prime}}{\left(1+w_{x}\right) \mathscr{H}}+\gamma^{2} \frac{a^{2} \bar{\rho}_{c} \bar{\rho}_{x}}{\mathscr{H}^{2}} .
\end{gathered}
$$

Using equations (5), (9), (10) and (39), the numerical values of these coefficients can be calculated directly from the background solutions.

When $\gamma>0$, in the radiation dominated era one can assume $\frac{\gamma a \bar{\rho}_{x}}{\mathscr{H}} \ll 1, \frac{\gamma a \bar{\rho}_{c}}{\mathscr{H}} \gg 1$ (see the right panel of Figure 10. In this limiting case the dark energy has no effect on the evolution of other fractions and their perturbations. By applying equation 9, quantity $f$ can be approximated as

$$
f \approx-2-\frac{a \gamma \bar{\rho}_{x}}{\mathscr{H}}-\frac{\mathscr{H}^{\prime}}{\mathscr{H}^{2}}
$$

Keeping only the dominant terms, the equation $(35)$ reduced to

$$
\delta_{x}^{\prime \prime}+\mathscr{H} \frac{b+1}{1+w_{x}} \frac{\gamma a \bar{\rho}_{c}}{\mathscr{H}} \delta_{x}^{\prime}+3 \mathscr{H}^{2}\left(1-w_{x}\right)\left(3+\frac{b}{1+w_{x}} \frac{\gamma a \bar{\rho}_{c}}{\mathscr{H}}\right) \delta_{x}=\mathscr{H}^{2} \frac{b+1}{1+w_{x}}\left(\frac{\gamma a \bar{\rho}_{c}}{\mathscr{H}}\right)^{2} \delta_{c}
$$

Coefficients $A_{x}$ and $B_{x}$ are positive and catastrophic growth perturbations do not occurs. In order of magnitude, equation (45) implies the estimation

$$
\delta_{x} \sim \frac{\gamma a \bar{\rho}_{c}}{\mathscr{H}} \delta_{c}
$$

For example, the "standard" adiabatic condition $\delta \rho_{c} / \bar{\rho}_{c}^{\prime}=\delta \rho_{x} / \bar{\rho}_{x}^{\prime}$ taking into account the background equations (9), (10) in the radiation dominated era yields

$$
\delta_{x}=-\frac{\gamma a \bar{\rho}_{c}}{3 \mathscr{H}} \delta_{c} \propto \tau
$$

as for the adiabatic mode in the synchronous gauge $\delta_{c} \propto \tau^{2}$.

When $\gamma<0$, the coefficients $A_{x}, B_{x}$ can take large negative values in regime of very strong coupling if $\left|\gamma a \bar{\rho}_{c} / \mathscr{H}\right| \gg 1$. In scenarios with $b=1$ and $w_{x}(a)<-1 / 3$ at early times they are becoming both negative together under this condition. Hence such models suffer from the fast growth of non-adiabatic perturbations in early Universe. However, constraints on coupling with $b=0$ can be weakened, because in this case in some range of parameters $w_{0}, w_{1}$ the coefficient $B_{x}$ remains positive and increases in the strong coupling regime.

To verify the analytical conclusions we have modified the public available CAMB code [31]. The initial adiabatic conditions for all non-dark species are imposed the same as in the non-interacting case. The initial values of dark sector perturbations are taken in accordance with $\delta \rho_{c} / \bar{\rho}_{c}^{\prime}=\delta \rho_{x} / \bar{\rho}_{x}^{\prime}=\delta \rho_{r} / \bar{\rho}_{r}^{\prime}$. In the presence $\gamma_{x} \neq 0$ the cold dark mater rest frame and synchronous frame are not coincide and it is not possible to adopt the CAMB conventions $\theta_{c} \equiv 0$ consistently. In our numerical calculations the residual synchronous gauge freedom was fixed by choosing $\theta_{c}\left(\tau_{\text {in }}\right)=0$, where $\tau_{\text {in }}$ is the time moment of the initial conditions setting. The results are shown in Figure 2 

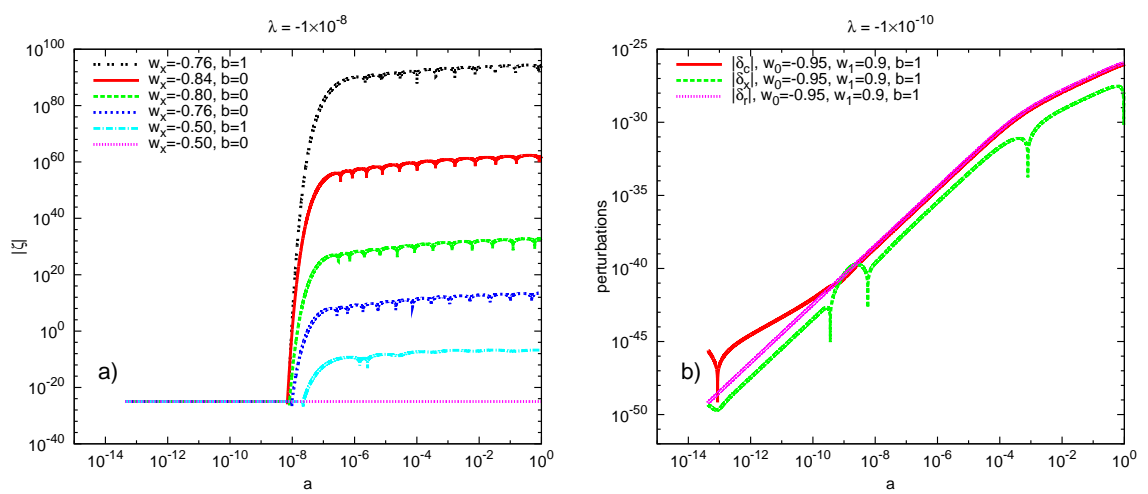

Fig. 2 a) The curvature perturbation $\zeta$ on super-Hubble scale in models with coupling 41 and constant dark energy equation of state $w_{x}<-1 / 3$. Growth of the non-adiabatic perturbations begins at the stage of the strong interaction. b)The density perturbation evolution in model with $w_{x}>-1 / 3$ in radiation dominated era. Initial dark sector conditions are set at $\tau_{i n}=2 \times 10^{-8} \mathrm{Mpc}$ by $\delta \rho_{c} / \bar{\rho}_{c}^{\prime}=\delta \rho_{x} / \bar{\rho}_{x}^{\prime}=\delta \rho_{r} / \bar{\rho}_{r}^{\prime}, \theta_{x}\left(\tau_{\text {in }}\right)=0$. In both cases the background densities corresponds to present values $\Omega_{x}=0.70, \Omega_{c}=0.2538, \Omega_{b}=0.0462$, the initial curvature perturbation $\zeta\left(\tau_{\text {in }}\right)$ equal to $1 \times 10^{-25}$ and comoving wave number is $k=7 \times 10^{-5} \mathrm{Mpc}^{-1}$.

\section{Special case.}

The special case of (22) is the interaction

$$
Q^{\mu}=\gamma \rho_{c} \rho_{x}\left(u_{c}^{\mu}-u_{x}^{\mu}\right)
$$

In the linear perturbation theory the velocity potential is a first order quantity, and hence the spatial components of 4-vector $Q^{\mu}$ are proportional to the product of the densities of both dark species and relative velocity. In components

$$
Q^{0}=0, \quad Q^{i}=\frac{\gamma \rho_{c} \rho_{x}}{a}\left(v_{c}^{, i}-v_{x}^{i}\right) .
$$

The background dynamics here is the same as at minimal coupling, but the perturbation evolution is different. The coefficients of second-order equation 35 for the large scale perturbations are

$$
\begin{gathered}
A_{x}=1-3 w_{x}-\frac{2 \mathscr{H}^{\prime}}{\mathscr{H}^{2}}+\frac{\gamma a \bar{\rho}_{c}}{\left(1+w_{x}\right) \mathscr{H}}-f \\
B_{x}=3\left(\left(1-w_{x}\right)\left(1-\frac{\mathscr{H}^{\prime}}{\mathscr{H}^{2}}-f\right)-\frac{w_{x} w_{x}^{\prime}}{\left(1+w_{x}\right) \mathscr{H}}+\frac{1-w_{x}}{1+w_{x}} \frac{\gamma a \bar{\rho}_{c}}{\mathscr{H}}\right) .
\end{gathered}
$$

Since now $\bar{\rho}_{c}=\rho_{c 0} a^{-3}$, we have $\left|\gamma a \bar{\rho}_{c} / \mathscr{H}\right| \gg 1$ in early Universe. Thus, at $\gamma<0$ the coefficients $A_{x}, B_{x}$ are both negative and there is a rapid growth of non-adiabatic perturbations in the radiation dominated era. At positive $\gamma$ the non-adiabatic growth of long-wavelength perturbations do not occurs. These analytical results are confirmed by numerical computations.

In the short-wave approximation the equations (30) - 34) can be combined into

$$
\begin{array}{r}
\delta_{x}^{\prime \prime}+\mathscr{H}\left(1-3 w_{x}-\frac{w_{x}^{\prime}}{\left(1+w_{x}\right) \mathscr{H}}+\frac{\gamma a \rho_{c}}{\left(1+w_{x}\right) \mathscr{H}}\right) \delta_{x}^{\prime}+k^{2} \delta_{x} \\
=\mathscr{H}^{2}\left(1+w_{x}\right)\left(\frac{3}{2}\left(\delta+3 \frac{\delta P}{\rho}\right)-\left(3-\frac{\gamma a \rho_{c}}{\left(1+w_{x}\right) \mathscr{H}}\right) \frac{\delta_{c}^{\prime}}{\mathscr{H}}-3 \frac{\theta_{c}}{\mathscr{H}}\right),
\end{array}
$$




$$
\begin{array}{r}
\delta_{c}^{\prime \prime}+\mathscr{H}\left(1+\frac{\gamma a \bar{\rho}_{x}}{\mathscr{H}}\right) \delta_{c}^{\prime} \\
=\mathscr{H}^{2}\left(\frac{3}{2}\left(\delta+3 \frac{\delta P}{\rho}\right)+\frac{\gamma a \bar{\rho}_{x}}{\left(1+w_{x}\right) \mathscr{H}}\left(3\left(1-w_{x}\right) \delta_{x}+\frac{\delta_{x}^{\prime}}{\mathscr{H}}\right)\right) .
\end{array}
$$

At positive $\gamma$ all coefficients in the left hand side of these equations are also positive, what excludes the presence of small scale adiabatic instabilities. For instance, at early times in the strong coupling regime with $\frac{a \gamma \bar{\rho}_{c}}{\mathscr{H}} \mathscr{H}^{2} \gg k^{2} \gg \mathscr{H}^{2}$ the first equation gives at leading order

$$
\delta_{x}^{\prime}=\left(1+w_{x}\right) \delta_{c}^{\prime} .
$$

Equations (52) and (53) take the same form as in the minimal coupling case under conditions $\frac{a \gamma \bar{\gamma}_{c}}{\mathscr{H}} \ll 1$ and $\frac{a \gamma \bar{\rho}_{x}}{\mathscr{H}} \ll 1$ respectively. In particular, when $\gamma \rightarrow 0$, the second one reduced to the standard growth equation.

\section{Conclusions.}

We examined the covariant generalization of the coupling (4). The evolution of perturbations is studied paying particular attention to the most favored interaction rate that is proportional to the product of dark matter and dark energy densities. It is shown that the models of the form (41) with $b=1$ and $w_{x}(\tau)<-1 / 3$ in radiation dominated era suffers from early time instabilities due fast growth of large scale non-adiabatic perturbations. Models with $w_{x}(\tau)>-1 / 3$ in radiation dominated Universe are free from this defect. Also an interesting coupling (48) with positive $\gamma$ is viable.

\section{References}

1. P. Astier et al., "The Supernova legacy survey: Measurement of omega(m), omega(lambda) and W from the first year data set", Astron. Astrophys., 447, 3148 (2006).

2. E. Komatsu et al., "Five-Year Wilkinson Microwave Anisotropy Probe (WMAP) Observations: Cosmological Interpretation", Astrophys. J. Suppl. 180, 330-376 (2009).

3. M. Tegmark et al., "Cosmological Constraints from the SDSS Luminous Red Galaxies", Phys. Rev. D74, 123507 (2006)

4. S. Weinberg,'The Cosmological Constant Problem”, Rev. Mod. Phys. 61, 1-23 (1989).

5. Mark B. Hoffman "Cosmological constraints on a dark matter dark energy interaction", arXiv:astro-ph/0307350.

6. C. Wetterich, "The cosmon model for an asymptotically vanishing time-dependent cosmological constant", Astron. Astrophys. 301, 321-328 (1995).

7. L. Amendola,'Scaling solutions in general non-minimal coupling theories", Phys. Rev. D60, 043501 (1999).

8. D.J. Holden and D. Wands,"Self-similar cosmological solutions with a non-minimally coupled scalar field",Phys. Rev. D61, 043506 (2000).

9. Glennys R. Farrar, and P. J. E. Peebles "Interacting Dark Matter and Dark Energy", Astrophys.J. 604, 1-11 (2004).

10. H. Ziaeepour, "Quintessence From The Decay of a Superheavy Dark Matter”, Phys. Rev. D69, 063512 (2004).

11. T. Koivisto "Growth of perturbations in dark matter coupled with quintessence", Phys. Rev. D72 043516 (2005)

12. R. Bean , E. E. Flanagan , I. Laszlo and M. Trodden, ”Constraining Interactions in Cosmology's Dark Sector", Phys. Rev. D78, 123514 (2008).

13. G. Caldera-Cabral, R. Maartens, L. A. Urena-Lopez, ”Dynamics of interacting dark energy”, Phys. Rev. D79, 063518 (2009). 
14. L.P. Chimento, A.S. Jakubi, D. Pavon and W. Zimdahl, "Interacting quintessence solution to the coincidence problem", Phys. Rev. D67, 083513 (2003).

15. M. Quartin, M.O. Calvao, S.E. Joras, R.R. Reis and I. Waga, ’Dark Interactions and Cosmological FineTuning", JCAP 0805, 007 (2008).

16. J. Valiviita, E. Majerotto and R. Maartens, "Large-scale instability in interacting dark energy and dark matter fluids", JCAP 0807, 020 (2008).

17. Jian-Hua He, B. Wang, E. Abdalla, "Stability of the curvature perturbation in dark sectors' mutual interacting models", Phys. Lett. B671, 139-145 (2009).

18. M.B. Gavela, D. Hernandez, L. Lopez Honorez, O. Mena and S. Rigolin, ”Dark coupling”, JCAP 0907, 034 (2009).

19. B. M. Jackson, A. Taylor, A. Berera, ”On the large-scale instability in interacting dark energy and dark matter fluids", Phys. Rev. D79, 043526 (2009).

20. G. Caldera-Cabral, R. Maartens, B. M. Schaefer, "The Growth of Structure in Interacting Dark Energy Models", JCAP 0907, 027 (2009).

21. J. Valiviita, E. Majerotto and R. Maartens, "Observational constraints on an interacting dark energy model", Mon. Not. Roy. Astron. Soc. 402, 2355-2368 (2010).

22. G. Mangano, G. Miele and V. Pettorino, "Coupled quintessence and the coincidence problem", Mod. Phys. Lett. A18, 831 (2003).

23. P. J. Steinhardt, "Cosmological Challenges for the 21st Century", in "Critical Problems in Physics", edited by V. L. Fitch, D. R. Marlow, and M. A. E. Dementi, p.123, Princeton U. Press, Princeton (1997)

24. I. Zlatev, L. Wang and P. J. Steinhardt "Quintessence, Cosmic Coincidence, and the Cosmological Constant", Phys. Rev. Lett. 82, 896-899 (1999).

25. Yin-Zhe Ma, Y. Gong, and X. Chen, "Couplings between holographic dark energy and dark matter", arXiv:0901.1215 [astro-ph].

26. M. Chevallier and D. Polarski, "Accelerating Universes with Scaling Dark Matter", Int. J.Mod. Phys. D10, 213 (2001)

27. H. Kodama and M. Sasaki , "Cosmological Perturbation Theory", Prog. Theor. Phys. Suppl. 78, 1 (1984)

28. Chung-Pei Ma, E. Bertschinger, "Cosmological Perturbation Theory in the Synchronous and Conformal Newtonian Gauges", Astrophys. J. 455, 7-25 (1995).

29. C. Gordon and W. Hu, "A Low CMB Quadrupole from Dark Energy Isocurvature Perturbations", Phys.Rev. D70 083003 (2004).

30. C. Gordon, D. Wands, B. A. Bassett and R. Maartens, ”Adiabatic and entropy perturbations from inflation", Phys. Rev. D63 023506 (2001).

31. A. Lewis, A. Challinor, and A. Lasenby, "Efficient computation of CMB anisotropies in closed FRW models", Astrophys. J., 538, 473-476 (2000). 